\title{
Assessing Medication Adherence and Healthcare Utilization and Cost Patterns Among Hospital-Discharged Patients with Schizoaffective Disorder
}

\author{
Sudeep Karve $\cdot$ Michael Markowitz $\cdot$ Dong-Jing Fu • \\ Jean-Pierre Lindenmayer • Chi-Chuan Wang • \\ Sean D. Candrilli • Larry Alphs
}

Published online: 23 April 2014

(C) The Author(s) 2014. This article is published with open access at Springerlink.com

\begin{abstract}
Background Hospital-discharged patients with schizoaffective disorder have a high risk of re-hospitalization. However, limited data exist evaluating critical post-discharge periods during which the risk of re-hospitalization is significant.

Objective Among hospital-discharged patients with schizoaffective disorder, we assessed pharmacotherapy adherence and healthcare utilization and costs during sequential 60-day clinical periods before schizoaffective disorder-related hospitalization and post-hospital discharge. Methods From the MarketScan ${ }^{\circledR}$ Medicaid database (2004-2008), we identified patients ( $\geq 18$ years) with a schizoaffective disorder-related inpatient admission. Study
\end{abstract}

Electronic supplementary material The online version of this article (doi:10.1007/s40258-014-0095-8) contains supplementary material, which is available to authorized users.

\section{S. Karve $(\square)$}

Germantown, MD, USA

e-mail: sudeepkarve@gmail.com

M. Markowitz

Cary, NC, USA

D.-J. Fu · L. Alphs

Janssen Scientific Affairs, LLC, Titusville, NJ, USA

J.-P. Lindenmayer

Psychopharmacology Research Unit, Manhattan Psychiatric

Center, New York University, New York, NY, USA

C.-C. Wang

Taipei, Taiwan

S. D. Candrilli

RTI Health Solutions, 200 Park Offices Drive/\#285, Research

Triangle Park, NC 27709, USA measures including medication adherence and healthcare utilization and costs were assessed during sequential preadmission and post-discharge periods. We conducted univariate and multivariable regression analyses to compare schizoaffective disorder-related and all-cause healthcare utilization and costs (in 2010 US dollars) between each adjacent 60 -day post-discharge periods. No adjustment was made for multiplicity.

Results We identified 1,193 hospital-discharged patients with a mean age of 41 years. The mean medication adherence rate was $46 \%$ during the 60 -day period prior to index inpatient admission, which improved to $80 \%$ during the 60-day post-discharge period. Following hospital discharge, schizoaffective disorder-related healthcare costs were significantly greater during the initial 60-day period compared with the 61- to 120-day post-discharge period (mean US $\$ 2,370$ vs US $\$ 1,765 ; p<0.001$ ), with rehospitalization $(36 \%)$ and pharmacy $(40 \%)$ accounting for over threefourths of the initial 60-day period costs. Compared with the initial 60-day post-discharge period, both all-cause and schizoaffective disorder-related costs declined during the 61- to 120-day post-discharge period and remained stable for the remaining post-discharge periods (days 121-365).

Conclusions We observed considerably lower (46\%) adherence during 60 days prior to the inpatient admission; in comparison, adherence for the overall 6-month period was $8 \%(54 \%)$ higher. Our study findings suggest that both short-term (e.g., 60 days) and long-term (e.g., 6-12 months) medication adherence likely are important characteristics to examine among patients with schizoaffective disorder and help provide a more holistic view of patients' adherence patterns. Furthermore, we observed a high rate of rehospitalization and greater healthcare costs during the initial 60-day period post-discharge among patients with schizoaffective disorder. Further research is 
required to better understand and manage transitional care after discharge (e.g., monitor adherence), which may help reduce the likelihood of rehospitalization and the associated downstream costs.

\section{Key Points for Decision Makers}

Short-term (e.g., 60 days) adherence coupled with long-term (e.g., 365 days) adherence to the prescribed therapy provides a holistic view of adherence patterns among the schizoaffective disorder patient population.

This study identifies the critical post-discharge period during which patients with schizoaffective disorder have significantly greater likelihood of rehospitalization and higher costs.

Decision makers should evaluate and consider the allocation of additional resources for post-discharge interventions (e.g., pharmacists/nurse counseling, developing post-discharge follow-up plans) which may help lower the cost and rehospitalization burden during the critical post-discharge period.

The poor medication adherence and substantial economic burden observed among patients with schizoaffective disorder highlight the need for conducting studies independently among patients with schizoaffective disorder rather than as part of a schizophrenia population.

\section{Introduction}

Schizoaffective disorder is a psychiatric disorder characterized by depressive, manic, and psychotic symptoms. The prevalence of schizoaffective disorder is estimated to be approximately one-third to one-sixth as common as schizophrenia (about $0.3 \%$ in the United States) [1, 2]. Even with lower prevalence than schizophrenia, findings from a recent US hospital discharge survey suggest that a slightly greater number of hospital-discharged patients had a schizoaffective disorder diagnosis (primary) than had schizophrenia [3]. In addition, persons with this diagnosis have been suggested to have a greater risk of suicide, substance abuse, and hepatic function abnormalities [4]. Patients with schizoaffective disorder often experience hallucinations, delusions which are characteristic of schizophrenia, as well as mood symptoms such as mania and depression that are associated with bipolar disorder. Literature suggests that age of onset for adult schizoaffective disorder spans age of onset of bipolar disorder and schizophrenia, with median age falling between the median age of schizophrenia and bipolar disorder [5]. Schizoaffective disorder is more common among females whereas schizophrenia is more common among males; an equal gender distribution is reported for bipolar disorder [5-8]. Overall, the overlap of symptoms of schizoaffective disorder with those of schizophrenia and bipolar disorder makes the clinical diagnosis difficult $[9,10]$ and the complex interplay of symptoms poses challenges to treatment which are frequently managed by polypharmacy. This increases the complexity of treatment with increased potential problems of adherence, drug-drug interactions, and cost of therapy.

As a chronic condition, schizoaffective disorder requires long-term pharmacologic treatment that includes acute treatment to manage symptom exacerbations, and maintenance therapy to lower the risk of relapse. Pharmacologic treatment generally includes antipsychotics used in combination with mood stabilizers or antidepressants $[1,11-$ 14]. Given that pharmacological treatment plays a key role in schizoaffective disorder management, it is important that patients receive continuous effective coverage with them. Patients with schizophrenia or schizoaffective disorder who use medication irregularly are approximately twice as likely to be rehospitalized and have $12 \%$ higher inpatient costs than patients who use their medication regularly [15].

Additionally, hospitalized patients with schizoaffective disorder have an increased likelihood of relapse and rehospitalization immediately following hospital discharge. The risk is even greater than that for patients with schizophrenia [16]. One study found that patients with schizoaffective disorder are 1.8 times more likely to experience a rehospitalization than patients with schizophrenia [17]. Among patients with schizoaffective disorder, increased risk of readmission following hospital discharge may lead to higher healthcare costs. In this study we examine healthcare utilization, and costs for patients with schizoaffective disorder over small (60-day), sequential periods during the high-risk post-discharge period.

\section{Methods}

\subsection{Study Design and Data Source}

This retrospective cohort study was conducted using MarketScan's Medicaid Multi-State (MMM) database from 2004 through 2008. This database contains information contributed from 11 US states. For confidentiality purposes, the states contributing data are not explicitly identified. The data include medical claims for services provided at various care settings (e.g., inpatient, outpatient, and emergency department [ER]) and outpatient pharmacy claims. Medical claims include details on physician diagnosis, date of service, length of stay (inpatient services), 
procedures performed, and information on payments made to a provider for services. Similarly, outpatient pharmacy claims include details on the generic name of the prescribed medication, national drug code, date of service, days' supply, and payment. In addition, the data also include details on patient demographic characteristics (e.g., age, gender) and health plan enrollment details (e.g., enrollment period, plan type, mental health coverage). The database employs a unique encrypted patient identifier that enables patients to be tracked longitudinally.

\subsubsection{Study Sample}

Patients from the MMM database with an inpatient admission primary diagnosis of schizoaffective disorder (International Classification of Diseases, 9th Revision, Clinical Modification [ICD-9-CM] diagnosis code 295.7x) during the period July 1, 2004 through December 31, 2007 were identified for this analysis. The date for the first observed inpatient admission was designated the index admission date,' and the date of discharge associated with the index admission was designated the 'index discharge date.' Patients were required to have a continuous Medicaid enrollment for up to 6 months before the index admission date (i.e., pre-index period), during the index hospitalization episode, and up to 12 months after the index discharge date (i.e., post-index period). Since the selection of patients with schizoaffective disorder was based on ICD-9-CM codes, any coding inaccuracies may lead to misclassification of patients. Thus, additional inclusion and exclusion criteria were applied to help improve the ascertainment of patients with schizoaffective disorder. Patients were required to have at least one schizoaffective disorder-related outpatient or physician office visit or at least two prescription claims for antipsychotics, antidepressants, or mood stabilizers. This may have been present anytime during the 6-month pre-index period or the 12-month postindex period. Patients were excluded if they had a claim with a diagnosis for other psychiatric conditions during the post-index period. The exclusion criterion varied, depending on the psychiatric condition. Patients with at least one primary diagnosis claim for bipolar disorder (ICD-9-CM codes 296.0x, 296.1x, 296.4x-296.8x, 301.1) or schizophrenia (ICD-9-CM code 295.xx [except 295.7x]) and patients with two or more primary diagnosis claims for unipolar disorder (ICD-9-CM codes 296.2x, 296.3x, 298.0x, 300.4, 311) during the 12-month post-index period were excluded. This criterion was applied to minimize the likelihood of misclassification of schizoaffective disorder with other psychiatric conditions.

Furthermore, to ensure that the selected index inpatient admission event was the first observed schizoaffective disorder-related inpatient admission and not a readmission, we excluded patients with an inpatient admission with a schizoaffective disorder diagnosis (primary or secondary) any time during the 6-month pre-index period (i.e., clean period). Patients were excluded if they were 18 years or younger at their index admission date. To be able to fully capture patients' health service utilization and costs, we excluded patients who were 65 years or older (i.e., Medicare-eligible patients) at follow-up end date (i.e., index discharge date plus 360 days) and patients who were eligible for both Medicare and Medicaid. Finally, patients without mental health and substance abuse coverage were excluded from this study.

\subsection{Study Measures}

\subsubsection{Medication Adherence}

Adherence to schizoaffective disorder-related medications (i.e., antipsychotics, antidepressants, and mood stabilizers) was assessed using the proportion of days covered (PDC) measure. PDC was calculated as:

PDC $=$ total days of drug availability (days' supply) in the period of evaluation $\div$ (number of days in the period of evaluation - number of days hospitalized during the period of evaluation)

When calculating the PDC, drug availability was assessed for each day of the study period. Given the multiple time points of evaluation (the 60-day increments in the pre-index and post-index periods), PDC was deemed more appropriate in calculating adherence rate than measuring the cumulative drug exposure as determined by the commonly used measure 'medication possession ratio' [18-20]. PDC was calculated for all schizoaffective disorder-related medications received during the follow-up period, irrespective of whether these medications were received as monotherapy or as combination therapy. Among patients receiving combination therapy (e.g., an antipsychotic and a mood stabilizer), as long they had evidence for one or more drugs on a day they were classified as being adherent for that day.

One of the limitations of inpatient claims data is that details regarding inpatient medication use are not available. To address this limitation, we assumed that patients were fully compliant with their prescribed drug regimens during an inpatient stay; we therefore subtracted hospitalization days from the denominator [18, 21]. Furthermore, our data did not include days' supply details for injectable drugs administered at a physician's office. Thus, in calculating PDC for depot antipsychotics administered at a physician's office, we used labeled dosage duration (i.e., risperidone 2 weeks, fluphenazine 3 weeks, and haloperidol 4 weeks) as a proxy for days' supply.

After calculating PDC, we categorized patients as adherent if their PDC value was 0.8 or greater and 
nonadherent if their PDC value was $<0.8$ [22-25]. Medication adherence was assessed for each 60-day interval in the pre- and post-index periods. We also assessed adherence for the overall 6-month pre-index and post-index periods and for the overall 12-month post-index period.

\subsubsection{Healthcare Utilization and Costs}

We assessed all-cause and schizoaffective disorder-related healthcare utilization and costs on a short-term, sequential basis for each 60-day interval in the 12-month post-index period. In addition, we assessed all-cause and schizoaffective disorder-related healthcare utilization and costs on a long-term basis during the 6-month pre-index and postindex periods and the overall 12-month post-index period. Since costs incurred during the index inpatient admission are neither part of the pre- nor the post-index period costs, they were not considered in calculating the pre-index and post-index period costs. Schizoaffective disorder-related healthcare utilization was defined as medical claims with a primary diagnosis code for schizoaffective disorder or outpatient pharmacy claims for schizoaffective disorderrelated medications, including antipsychotics, antidepressants, and mood stabilizers. Both all-cause and schizoaffective disorder-related healthcare utilization and costs were assessed and reported by care settings, including inpatient, outpatient, physician office, ER, pharmacy, and other ancillary care. All costs outcomes were adjusted to 2010 US dollars using the medical care component of the Consumer Price Index.

Finally, for each patient, we calculated per-day costs for an inpatient episode as:

Total costs for an inpatient episode $\div$ length of stay

For patients whose inpatient stay spanned two or more follow-up periods (e.g., 0- to 60-day and 61- to 120-day periods), we calculated length of stay for each period. Further, the length of stay in each follow-up period was multiplied by per-day cost to provide total inpatient costs for each follow-up period.

\subsection{Statistical Analysis}

The key explanatory variables considered for this study were indicators for the 60-day follow-up periods. All study measures were compared between two contiguous 60-day follow-up periods (e.g., 0-60 and 61-120 days), with the former period being the reference group (e.g., 0-60 days). Since we wanted to assess if healthcare utilization and costs varied (increases or decreases) over time we considered contiguous 60-day follow-up periods for comparisons rather than anchoring the comparisons to a single period (e.g., 0-60 days). Other covariates considered were age at the index admission date, gender, race, plan type, basis of
Medicaid eligibility, index hospitalization discharge status, and baseline comorbidity burden (assessed using the Charlson Comorbidity Index score) [26]. The data did not include information on disease severity. Prior studies conducted among patients with mental health disorders have used prior costs as a proxy measure for disease severity and predictor of future healthcare costs [27-29]. Therefore, we used pre-index costs, calculated as the sum of all healthcare costs incurred during the 6-month pre-index period as a covariate in the regression analyses. Patients were categorized as 'high-cost users' (pre-index period costs $\geq 75$ th quartile) and 'non-high-cost users' (i.e., pre-index period costs $<75$ th quartile) based on the pre-index period costs. The 75th quartile costs cut-off used was US\$15,650.

Descriptive statistics, including means and standard deviations (SDs), were reported for continuous variables, while counts and percentages were reported for categorical variables. For unadjusted analyses, we used paired $t$ tests (i.e., continuous measures) and McNemar's test (i.e., categorical measures) to compare the outcome measures between each adjacent study period.

Healthcare utilization and costs were also compared between each contiguous follow-up period, using covariate-adjusted (i.e., baseline demographic and clinical characteristics) regression models. Count data outcomes (e.g., number of unique hospitalizations) were compared using repeated-measure Poisson or negative binomial regression models. For each count data outcome, selection of a Poisson or negative binomial regression model was made by model fit, which was determined by the Pearson chisquare test. The incident rate ratio (IRR) generated using the Poisson or negative binomial regression model represents the increased or decreased rate of healthcare utilization during a follow-up period relative to the reference follow-up period. Finally, healthcare costs were compared using repeated-measure generalized linear models with a log-link function and gamma distribution. A generalized linear model was chosen over a log-transformed ordinary linear regression because it estimates covariate-adjusted predicted mean costs on a dollar scale and thus avoids potential biases resulting from the Duan smearing method for retransforming [30-32]. In our study sample, only a small number of patients $(<1.5 \%)$ experienced an ER event during the follow-up; thus, we could not conduct covariate-adjusted regression analyses to compare ER utilization and costs between each continuous follow-up period. No adjustment was made for multiplicity.

\section{Results}

After applying the study's inclusion and exclusion criteria, 1,193 patients were included in our final sample (Online 
Resource 1 includes a figure depicting the study periods [Supplementary Figure 1] and an attrition chart [Supplementary Figure 2] which documents how this sample was identified). Baseline patient characteristics are shown in Table 1 . The mean age of these patients was 41 years, $61 \%$ were female, and $48 \%$ were white. Sixty-eight percent of these patients had fee-for-service coverage and $92 \%$ were 'discharged to home for self-care' following an inpatient admission. Approximately $25 \%$ of patients had high healthcare costs (high costs cut-off used was US $\$ 15,650)$ in the 6-month pre-index period.

\subsection{Medication Adherence}

Table 2 summarizes adherence to schizoaffective disorderrelated medications in the 6-month pre-index and the 12-month post-index periods. During the pre-index period, medication adherence rates as measured by PDC declined from 0.65 (SD 0.38) in the 182- to 121-day period to 0.46 (SD 0.45) in the 60- to 0-day period. Compared with the initial 60-day pre-index period, a higher adherence rate was observed in the initial 60-day post-index period (PDC 0.80; SD 0.27). However, the adherence rate dropped to 0.63 (SD 0.40 ) in the 61- to 120-day post-index period and remained relatively stable in the 121- to 180-day post-index periods (PDC 0.62; SD 0.42). When looking at the longer term adherence, on average, the PDC measured during the 6-month post-index period was higher than the overall PDC measured during the 6-month pre-index period (0.69 vs 0.54). Similarly, we observed a higher percentage of patients who adhered to their treatment during the 6-month post-index period than during the 6-month pre-index period (51 vs $37 \%)$.

\subsection{Unadjusted Healthcare Utilization and Costs}

We observed greater schizoaffective disorder-related and all-cause healthcare utilization and costs during the initial 0 - to 60-day post-discharge period compared with other follow-up periods (Fig. 1). Thus, the results presented here primarily focus on comparing healthcare utilization for the two initial post-discharge periods (i.e., 0- to 60-day and 61to 120-day periods) (Figs. 2, 3). However, data for all study periods are presented in tabular format (Online Resource 1-Supplementary Tables 1 and 2).

\subsubsection{Unadjusted Schizoaffective Disorder-Related Utilization and Costs}

We observed a greater percentage of patients with at least one schizoaffective disorder-related encounter in the initial 0 - to 60-day post-index period than in the 61- to 120-day post-index period (82 vs $72 \% ; p<0.001$ ). A significantly
Table 1 Patient characteristics

\begin{tabular}{|c|c|c|}
\hline & $N$ (mean) & $\%(\mathrm{SD})$ \\
\hline Total sample & 1,193 & 100.00 \\
\hline \multicolumn{3}{|l|}{ Sex } \\
\hline Male & 460 & 38.56 \\
\hline Female & 733 & 61.44 \\
\hline \multicolumn{3}{|c|}{ Age (years) at index admission date ${ }^{a}$} \\
\hline Mean (SD) & 40.7 & $(11.84)$ \\
\hline \multicolumn{3}{|l|}{ Age distribution (years) } \\
\hline $18-24$ & 142 & 11.90 \\
\hline $25-34$ & 243 & 20.37 \\
\hline $35-44$ & 308 & 25.82 \\
\hline $45-54$ & 352 & 29.51 \\
\hline $55-64$ & 148 & 12.41 \\
\hline \multicolumn{3}{|l|}{ Race/ethnicity } \\
\hline White & 576 & 48.28 \\
\hline Black & 508 & 42.58 \\
\hline Hispanic & 6 & 0.50 \\
\hline Other & 103 & 8.63 \\
\hline \multicolumn{3}{|l|}{ Health coverage } \\
\hline Fee-for-service & 817 & 68.48 \\
\hline Capitated & 376 & 31.52 \\
\hline \multicolumn{3}{|l|}{ Basis of medicaid eligibility } \\
\hline Blind/disabled individual & 1,085 & 90.95 \\
\hline $\begin{array}{l}\text { Adult (not based on unemployed } \\
\text { status) }\end{array}$ & 80 & 6.71 \\
\hline $\begin{array}{l}\text { Child (not child of unemployed } \\
\text { adult, not foster-care child) }\end{array}$ & 17 & 1.42 \\
\hline Other $^{\mathrm{b}}$ & 11 & 0.92 \\
\hline \multicolumn{3}{|l|}{ Hospital discharge status } \\
\hline Discharged to home self-care & 1,097 & 91.95 \\
\hline Transfer to SNF & 13 & 1.09 \\
\hline Transfer to other facility & 39 & 3.27 \\
\hline Left against medical advice & 8 & 0.67 \\
\hline Other alive status & 1 & 0.08 \\
\hline Not yet discharged/transferred & 10 & 0.84 \\
\hline Missing/unknown & 25 & 2.10 \\
\hline
\end{tabular}

Pre-index period ${ }^{\mathrm{c}}$ all-cause healthcare costs (USD)

Mean (SD)

US\$11,577.20 (US\$13,324.09)

High-cost users

Non-high cost (i.e., pre-index $\quad 899 \quad 75.36$

period costs $<75$ th quartile)

High cost (i.e., pre-index period $294 \quad 24.64$ costs $\geq 75$ th quartile)

Pre-index Period Charlson Comorbidity Index Score

Mean (SD) $\quad 1.09 \quad$ (1.71)

$S D$ standard deviation, $S N F$ skilled nursing facility

${ }^{a}$ The date for the first observed schizoaffective disorder-related inpatient admission will define the index admission date

b 'Other' includes foster-care child, aged individual, and eligibility status unknown

c The 6-month period before the index admission date defines the pre-index period 
Table 2 Adherence $^{\mathrm{a}}$ to schizoaffective disorder-related medications ${ }^{\mathrm{b}}$

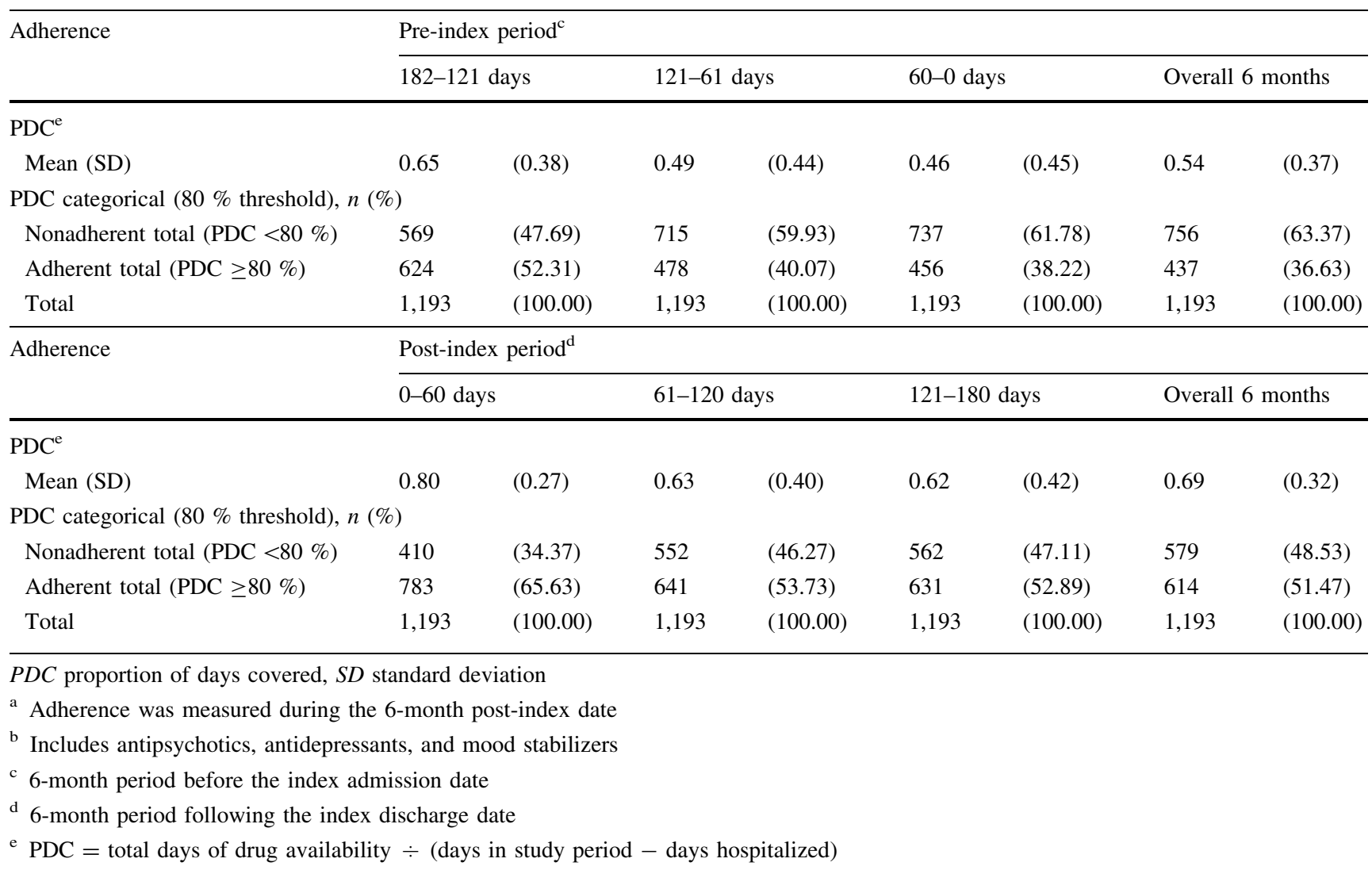

greater proportion of patients had at least one schizoaffective disorder-related prescription filled during the initial 60 -day post-index period than in the 61- to 120 -day postindex period (90 vs $78 \% ; p<0.001$ ). Other care settings with significantly greater schizoaffective disorder-related utilization during the initial 60-day period than in the 61- to 120-day period included inpatient services $(p<0.001)$, office visits $(p=0.02)$, and other ancillary care $(p<0.001)$. Schizoaffective disorder-related healthcare utilization declined and remained stable after the initial 60 -day post-index period.

Schizoaffective disorder-related healthcare costs (mean US\$2,370 vs US\$1,765; $p<0.001$ ) were significantly greater in the initial 0 - to 60-day post-index period than in the adjacent 61- to 120-day post-index period. The primary drivers of schizoaffective disorder-related costs in the 0- to 60-day post-index period were rehospitalization (mean US\$860; SD US\$3,923) and pharmacy (mean US\$954; SD US\$926). Schizoaffective disorder-related costs declined and remained stable after the initial 60-day post-index period (range of means US\$1,523-US\$1,764). No significant differences in schizoaffective disorder-related costs were observed in care settings including outpatient, physician office, and inpatient for the remaining post-index periods (all $p$ values $>0.05$ )
Finally, we observed significantly greater schizoaffective disorder-related mean total medical costs during the 6-month post-index discharge period compared with the 6-month pre-index period (US\$5,658 vs US\$2,925; $p<0.001)$.

\subsubsection{Unadjusted All-Cause Utilization and Costs}

A significantly greater percentage of patients had an allcause hospitalization (15.9 vs $10.1 \% ; p<0.001)$ and greater mean [SD] hospitalization costs (US\$1,521 [US\$5,912] vs US\$961 [US\$3,869]; $p<0.001)$ during the initial 0 - to 60-day post-discharge period compared with the 61- to 120-day post-discharge period. We also observed greater all-cause costs in pharmacy (US $\$ 1,567$ [US $\$ 1,314]$ vs US\$1,327 [US\$1,267]; $p<0.001)$ and hospital outpatient (US\$240 [US\$1,360] vs US\$167 [US\$734]; $p=0.016$ ) care settings during the 0 - to 60-day post-index discharge period compared with the 61- to 120-day postdischarge period. Following the initial 0- to 60-day postdischarge period, all-cause total medical costs (US\$5,277 [US\$7,649]) declined during the 61- to 120-day post-discharge period (US\$4,310 [US\$6,033], $p<0.001$ ) and remained stable during the remaining 60-day post-index discharge periods (range of means US\$3,998-US\$4,601). 


\section{A All Cause Costs}

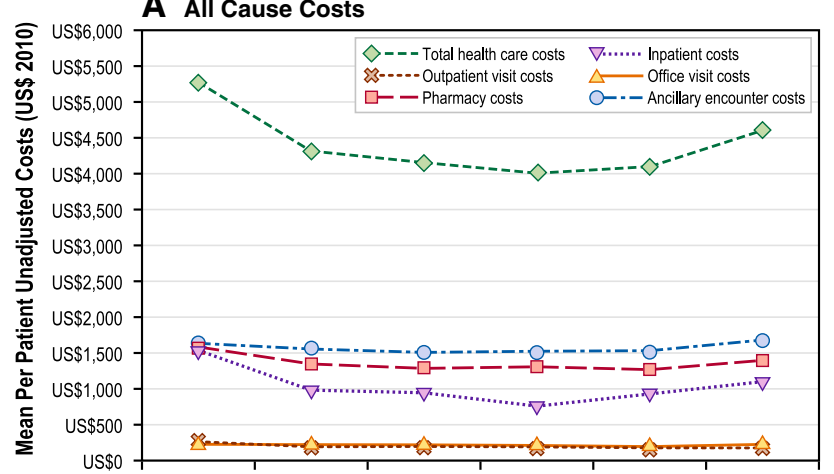

B Schizoaffective Disorder-related Costs

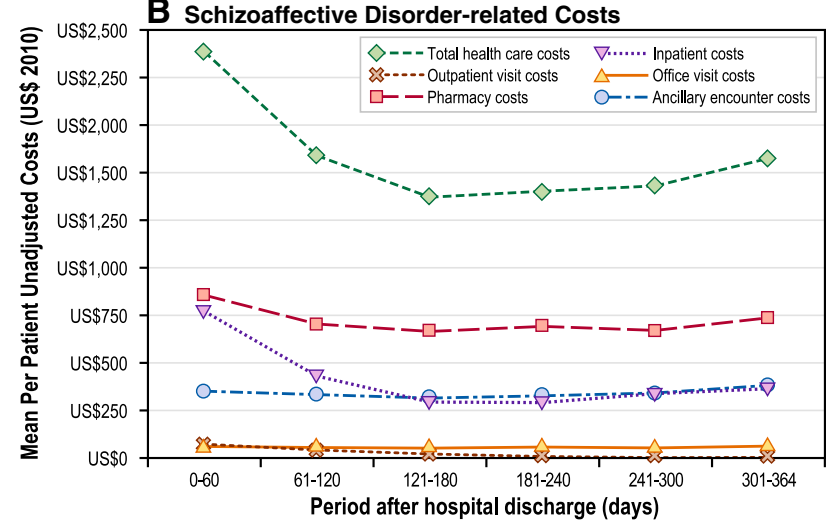

Fig. 1 Overall follow-up period unadjusted all-cause and schizoaffective disorder-related healthcare costs by care setting (the 12-month period following the index discharge date defines the post-index period)

Finally, all-cause total medical costs were significantly greater during the 6-month post-discharge date period compared with the 6-month pre-index period (US\$13,732 [US\$16,953] vs US\$11,462 [US\$13,132]; $p<0.001)$.

\subsection{Covariate-Adjusted Healthcare Utilization and Costs}

Figure 2 presents risk-adjusted IRRs for all-cause and schizoaffective disorder-related healthcare utilization, by care settings, for the 0- to 60-day and the 61- to 120-day post-index discharge periods. Similarly, Fig. 3 presents covariate-adjusted predicted all-cause and schizoaffective disorder-related healthcare costs, by individual care settings, for the 0- to 60-day and the 61- to 120-day post-index discharge periods. Risk-adjusted IRRs for all-cause and schizoaffective disorder-related healthcare utilization, by care settings, for the remaining follow-up periods are presented in Online Resource 1-Supplementary Table 3. Finally, the Online Resource 1-Supplementary Table 4 presents detailed regression model results for two outcome measures including schizoaffective disorder-related prescription drug costs and schizoaffective disorder-related prescription drug count.

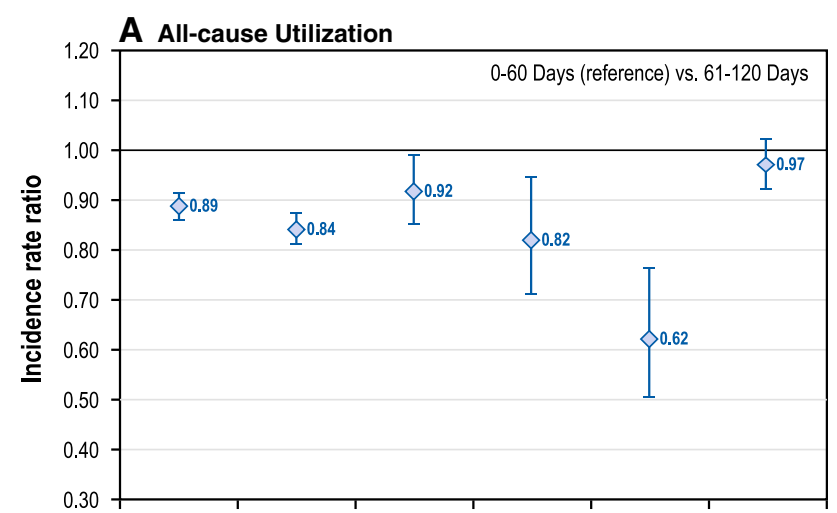

B Schizoaffective Disorder-related Utilization

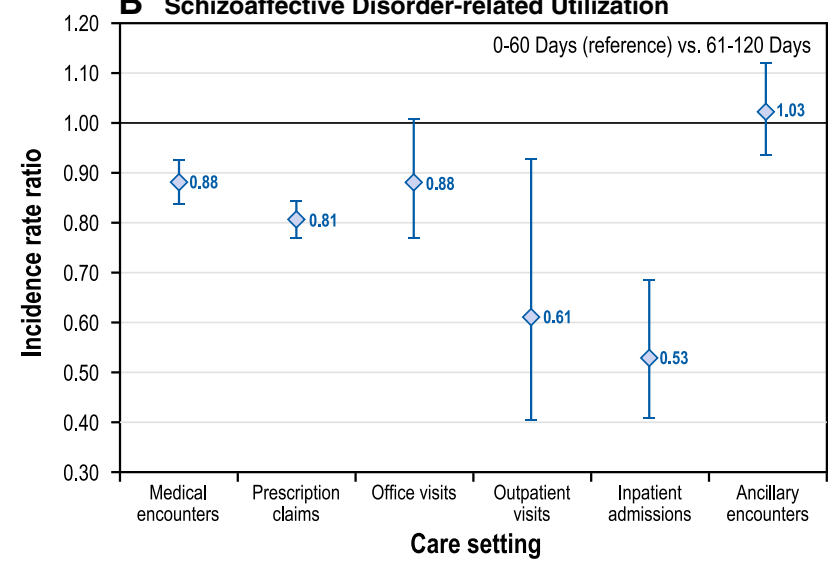

Fig. 2 Follow-up period (0-60 days vs 61-120 days) risk-adjusted incident rate ratios for all-cause and schizoaffective disorder-related health care utilization, by care setting (incident rate ratios based on negative binomial or Poisson regression models, adjusted for study period and other relevant covariates [i.e., gender, race, age, Charlson Comorbidity Index score, healthcare plan type, discharge status, antipsychotic adherence, and pre-index period healthcare cost]). Graph presents incident rate ratios and corresponding $95 \%$ confidence intervals comparing care setting-specific utilization rates during the $61-120$ and $0-60$ day (reference group) post-discharge periods

\subsubsection{Covariate-Adjusted Schizoaffective Disorder-Related Utilization and Costs}

Consistent with the unadjusted results, the adjusted results illustrate that the rates of service use for schizoaffective disorder-related pharmacy (IRR 0.81; confidence interval [CI] $0.77-0.85 ; p<0.001$ ), outpatient (IRR $0.61 ; 95 \% \mathrm{CI}$ $0.40-0.93 ; p=0.022$ ) and inpatient (IRR $0.53 ; 95 \% \mathrm{CI}$ $0.41-0.69 ; p<0.001)$ services use were significantly lower in the 61- to 120-day post-index period than in the initial 60-day post-index period. However, we did not observe significant differences in schizoaffective disorder-related inpatient utilization rate for the remaining post-index discharge periods relative to its immediate predecessor period: 121-180 days (IRR 0.79; $p=0.149$; compared with 61-120 days), $181-240$ days (IRR 0.96; $p=0.871$; compared with 121-180 days), 241-300 days (IRR 1.05; $p=0.767$; compared with 181-240 days) and 301-364 days 


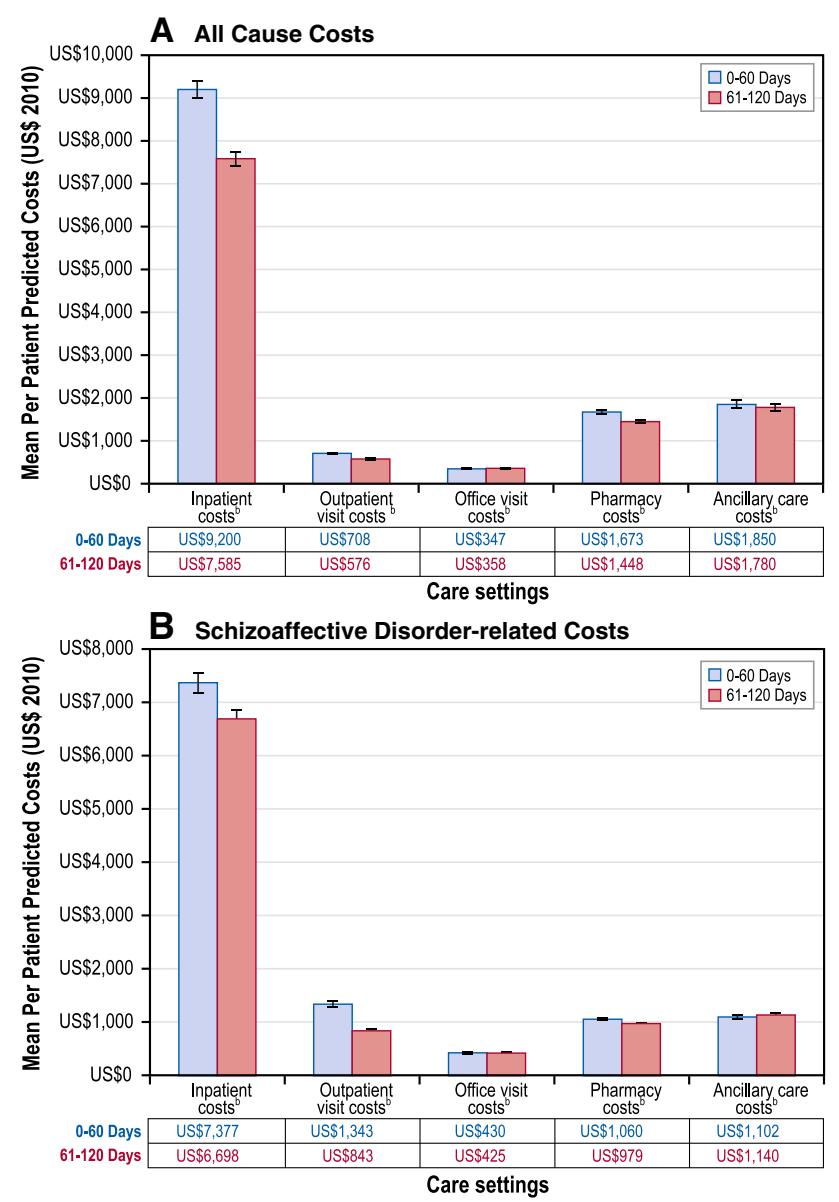

Fig. 3 Follow-up period risk-adjusted all-cause and schizoaffective disorder-related healthcare costs (predicted costs estimates and corresponding $95 \%$ confidence intervals based on generalized linear models, and corresponding $p$ values based on paired $t$ tests), by care setting. $p<0.001$

(IRR 1.07; $p=0.694$; compared with 241-300 days) (Online Resource 1-Supplementary Table 3). Schizoaffective disorder-related costs were lower across care settings, including pharmacy, physician office, hospital outpatient, and inpatient care, during the 61- to 120-day post-index discharge period compared with the 0 - to 60-day post-index discharge period (Fig. 3).

\subsubsection{Covariate-Adjusted All-Cause Utilization and Costs}

The utilization rate was $16 \%$ lower for all-cause pharmacy services (IRR 0.84; $95 \%$ CI 0.81-0.87; $p<0.001$ ) and $38 \%$ lower for all-cause inpatient admissions (IRR 0.62; $95 \%$ CI $0.51-0.76 ; p<0.001)$ in the 61 - to 120 -day postindex period compared with the 0 - to 60-day post-index period. However, all-cause inpatient utilization rates did not differ for the remaining post-index discharge periods: 121-180 days (IRR 0.70; $p=0.338$; compared with 61-120 days), $181-240$ days (IRR 0.89; $p=0.351$; compared with 121-180 days), 241-300 days (IRR 1.18; $p=0.163$; compared with 181-240 days) and 301-364 days (IRR 1.09; $p=0.430 ;$ compared with 241-300 days). After adjusting for covariates, the predicted all-cause costs were significantly lower for various care settings, including pharmacy (US\$1,448 vs US\$1,673; $p<0.001$ ), hospital outpatient (US\$576 vs US\$708; $p<0.001$ ), and inpatient (US\$7,585 vs US\$9,200; $p<0.001)$ costs during the 61- to 120-day post-index discharge period than during the 0- to 60-day post-index discharge period.

\section{Discussion}

Our study documents medication adherence patterns, healthcare utilization, and healthcare costs at sequential, clinically relevant, 60-day periods before an inpatient admission and post-hospital discharge among Medicaidenrolled patients with schizoaffective disorder. Adherence to prescribed medications declined during each of the 60 -day pre-index periods with the lowest rate observed during the 60-day period immediately prior to the inpatient admission. In contrast, adherence to schizoaffective disorder-related medications was highest during the 60-day period immediately following hospital discharge. Similarly, schizoaffective disorder-related costs and utilization were significantly higher during the initial 0- to 60-day post-discharge period compared with the 61- to 120-day post-discharge period.

Previous studies have suggested low adherence is associated with an increased risk of relapse and rehospitalization among patients with schizophrenia and schizoaffective disorder [15, 16, 33]. Typically, administrative claims-based studies assessing therapy adherence among patients with psychiatric conditions have focused on measuring long-term (e.g., 365 days) adherence [34-36]. In general, long-term adherence is an important measure of patient behavior towards the prescribed therapy and lower adherence rates $(<80 \%)$ have been shown to be associated with higher healthcare utilization and costs [37-39].

An important limitation of long-term adherence assessment using measures such as medication possession ratio (MPR) is that the entire adherence assessment period is equivalently weighted. Limiting the assessment of adherence to long periods without understanding the details around smaller intervals during which a patient may be non-adherent to the prescribed therapy reduces our ability to interpret the data. Two scenarios may help to clarify. Scenario 1: Patient A refills a 30-day supply of antipsychotic medication ten times in 1 year and with a 7-day gap between each refill, resulting in an annual adherence rate of $82 \%$ (300 days of drug on hand/365 days); Scenario 2: 
Patient B refills a 30-day supply of antipsychotic medication ten times in 1 year and with a 65-day gap after the last refill, also resulting in an annual adherence rate of $82 \%$ (300 days of drug on hand/365 days). Although both patients have the same number of days without drug annually, Patient A's poor adherence is distributed throughout the 12-month period, whereas that for Patient B is concentrated after the last prescription refill. Patient $\mathrm{B}$ with the concentrated gap is potentially at greater risk of having a relapse than Patient A. Thus, further studies evaluating the impact of concentrated and dispersed gaps in prescribed therapy and relapse rate are required. In this study we present both long- (6 months) and short-term (60-day period) adherence. The mean PDC for 6 months prior to inpatient admission was $54 \%$ whereas mean PDC during the 60-day period to the inpatient admission was $46 \%$. Thus, a more detailed analysis of adherence including short- and long-term timeframes should help understand patients' adherence behavior rather than only using short- or long-term adherence measures. Furthermore, short-term adherence may also help better identify patients at risk for relapse and needs to be studied further.

Among the selected patients, adherence to schizoaffective disorder-related pharmacotherapy generally declined over time during the period prior to the index inpatient admission. Patients with schizoaffective disorder are often managed by a combination of antipsychotics, antidepressants, and mood stabilizers, and patients using multiple medications within these classes may require regular blood level testing. The complexity and burden of the treatment in persons whose symptoms are associated with cognitive difficulties and apathy may lead to treatment overload and neglect of the treatment plan provided by their physician. The consequent loss of adequate exposure to therapy is likely to lead to relapse and hospitalization.

Another important finding of this study was the identification of the period of greatest risk for rehospitalization in these patients as the 60 days immediately following discharge. In addition, the total schizoaffective disorderrelated costs during this 60-day interval were more than $30 \%$ greater than the costs in any of the subsequent 60 -day post-discharge periods.

The increased risk of rehospitalization observed during this interval may be related to incomplete stabilization while hospitalized and to inadequate timely follow up immediately after discharge [16, 17, 40-42]. Several studies have found that failure to achieve such timely follow-up care is linked to an increased risk of rehospitalization [43, 44]. In this study, only $15 \%$ of patients had a schizoaffective disorder-related follow-up physician office visit during the 60-day post-discharge period. This suggests that one way to reduce high post-index costs and reduce the risk of readmission is to improve patients' transition from an inpatient setting to an outpatient setting. It has been reported that better continuity of care among patients with severe mental illness was associated with reduced hospital costs, improved quality of life and functioning, and reduced severity of symptoms [45, 46]. Thus, tailored management and treatment strategies during the first 60 days after hospitalization may be of particular importance. Supporting this concept, the proposed Healthcare Effectiveness Data and Information Set (HEDIS) health plan has set assessment of the rate of 7- and 30-day postdischarge follow-up outpatient visits as a performance measure for patients with schizophrenia or schizoaffective disorder [47, 48].

Overall, this study has several unique attributes. First, this study used a multi-state (11 states) Medicaid database and thus helped understand adherence patterns and resource utilization patterns across a geographically diverse population of Medicaid enrollees diagnosed with schizoaffective disorder. Furthermore, Medicaid is the largest payer for mental health care and facing declining funding and thus, measures to identify high risk and high utilizers of mental health services are necessary [49-52]. Secondly, our study applied several criteria to ensure that coding for schizoaffective disorder was captured as accurately as possible. Thirdly, our study findings indicate that the measures assessed in our study including short-term and long-term adherence and utilization over smaller sequential periods can serve as useful tools to identify high-risk patients who can be targeted for interventions to lower the readmission risk and the associated downstream costs. Moreover, these measures can be applied to other mental health conditions (e.g., schizophrenia, bipolar disorder) and using the low-cost administrative claims data, which are generated and provided by the Centers for Medicare and Medicaid on an annual basis.

However, several limitations should be considered in interpreting the study findings. First, the study was conducted using Medicaid claims data. Although there are not obvious differences in the patients in this set compared with others, it remains to be seen if these findings will generalize to uninsured populations or to individuals covered under commercial or public plans (e.g., Medicare). In addition, we excluded patients with dual eligibility (i.e., Medicare and Medicaid) and patients 65 years or older since these patients are primarily covered by Medicare, and Medicaid claims data may not fully capture health service utilization and costs for these patients. Thus, the study findings cannot be extrapolated to elderly patients with schizoaffective disorder. Furthermore, the inclusion and exclusion criteria used to select the study cohort may limit the generalizability of the results. For example, in order to ensure that each selected patient has a uniform length of follow-up data, we required patients to have continuous 
Medicaid enrollment during the study period. Thus, the selected study population excluded more severely ill patients treated in long-term settings. Additionally, our adherence measure was based on pharmacy claims, not on actual medication consumption. A key assumption when using this adherence measure is that the prescriptions that were filled were consumed as scheduled. This is not likely fully accurate in real world practice. Nevertheless, PDC remains a validated proxy for true adherence and represents one of the most commonly used approaches [18-20]. Furthermore, we assessed adherence to overall schizoaffective disorder-related therapy and not individual drugs among patients receiving two or more drugs simultaneously. Thus, among patients receiving combination therapy (e.g., an antipsychotic and a mood stabilizer), as long they had evidence for one or more drugs on a day they were classified as being adherent for that day. This was based on the assumption that patients are using some drug to manage the condition. The limitation of this approach is that among patients required to take multiple medications, adherence may be overstated if all drugs in the combination are necessary every day to confer a therapeutic benefit.

We assessed patient's age at index admission date and considered the commonly used age cut-off (i.e., $\geq 18$ years) to define adults in studies conducted using Medicaid databases including the MarketScan Multi-State database [5356]. The 17 patients with basis of eligibility as 'Child (not child of unemployed adult, not foster-care child)'that was assessed at the index admission date can possibly be from Medicaid states that consider 21 years as the age cut-off to define adults. However, we do not have information on the states contributing to the database and thus the age cut-off used by these states to define adults and children could not be determined. In addition, because patients' out-of-pocket costs and indirect costs, such as productivity losses and caregiver costs, were not available in the dataset used for these analyses, only direct medical costs paid by Medicaid were assessed in this study. All costs measures reported in this study are based on US\$ 2010, which was the most current complete year for which medical care component of the US Consumer Price Index was available at the time that this study was conducted. Finally, we were unable to control for several factors that may affect adherence, healthcare utilization, and costs (e.g., educational level and medication-related adverse events), as this information was unavailable in our data.

\section{Conclusions}

Overall, the lack of reliable diagnostic criteria often makes diagnosis of schizoaffective disorder a challenge which may explain the limited number of studies on schizoaffective disorder. A recent study reports that in the last 2 decades over 5,300 studies related to psychiatric conditions (bipolar disorder, schizophrenia, schizoaffective disorder) were published, of which $\sim 1 \%$ related to schizoaffective disorder [57]. Our study findings indicate that a considerable number of Medicaid enrollees had a schizoaffective disorder-related inpatient admission during the study period. Thus, understanding medication adherence and resource utilization independently among patients with schizoaffective disorder is important. In contrast, several previously published administrative claims-based studies have not considered these patients independent from the ones diagnosed with schizophrenia and have often included patients diagnosed with schizoaffective disorder as part of a schizophrenia cohort [58-62]. Our study helps address this limitation by evaluating medication adherence and resource utilization patterns across 60-day periods before and after hospitalization specifically among patients with schizoaffective disorder. We observed greater inpatient and pharmacy utilization in the initial 60-day postdischarge period than in any other 60-day intervals following discharge. Overall, findings from this study may help identify high-risk, high-cost patients and aid in designing interventions that may reduce the likelihood of hospital admissions and reduce costs associated with the care of schizoaffective patients.

Acknowledgments Source(s) of support Funding for this study was provided by Janssen Scientific Affairs, LLC, Titusville, NJ, USA. The publication of this study's results is not contingent on the sponsor's approval or censorship of the manuscript.

Conflict of interest Dong-Jing Fu, and Larry Alphs are employees of Janssen Scientific Affairs, LLC. At the time of conduct of this study Michael Markowitz was an employee of Janssen Scientific Affairs, LLC. Sean Candrilli is an employee of RTI Health Solutions, an independent contract research organization that received research funding from Janssen Scientific Affairs, LLC, for this study. JeanPierre Lindenmayer is a psychiatrist at the Psychopharmacology Research Unit, Manhattan Psychiatric Center, New York University. At the time of conduct of this study Sudeep Karve and Chi-Chuan Wang were employees of RTI Health Solutions.

Contributors' roles Michael Markowitz, Sudeep Karve, Dong Jing Fu, Jean-Pierre Lindenmayer, Sean D. Candrilli, and Larry Alphs were the primary developers of the study design. Sudeep Karve and Chi-Chuan Wang had full access to all the data in the study and take responsibility for the integrity of the data and the accuracy of the data analysis. Sudeep Karve, Chi-Chuan Wang, and Sean Candrilli led all statistical analyses. Sudeep Karve and Chi-Chuan Wang also drafted the manuscript text and assisted in interpreting the findings. Michael Markowitz, Dong Jing Fu, Jean-Pierre Lindenmayer, Sean D. Candrilli, and Larry Alphs assisted in interpreting the study findings and drafting the manuscript text; they also served as the primary reviewers of the manuscript. All authors were responsible for approving the manuscript and its contents.

Open Access This article is distributed under the terms of the Creative Commons Attribution Noncommercial License which 
permits any noncommercial use, distribution, and reproduction in any medium, provided the original author(s) and the source are credited.

\section{References}

1. Olfson M, Marcus SC, Wan GJ. Treatment patterns for schizoaffective disorder and schizophrenia among Medicaid patients. Psychiatr Serv. 2009;60(2):210-6.

2. Perälä J, Suvisaari J, Saarni SI, et al. Lifetime prevalence of psychotic and bipolar I disorders in a general population. Arch Gen Psychiatry. 2007;64(1):19-28.

3. DeFrances CJ, Cullen KA, Kozak LJ. National Hospital Discharge Survey: 2005 annual summary with detailed diagnosis and procedure data. Vital Health Stat. 2007;165:1-209.

4. Cheniaux E, Landeira-Fernandez J, Lessa Telles L, et al. Does schizoaffective disorder really exist? A systematic review of the studies that compared schizoaffective disorder with schizophrenia or mood disorders. J Affect Disord. 2008;106(3):209-17.

5. Abrams DJ, Rojas DC, Arciniegas DB. Is schizoaffective disorder a distinct categorical diagnosis? A critical review of the literature. Neuropsychiatr Dis Treat. 2008;4(6):1089-109.

6. Diflorio A, Jones I. Is sex important? Gender differences in bipolar disorder. Int Rev Psychiatry. 2010;22(5):437-52.

7. Ketter TA. Diagnostic features, prevalence, and impact of bipolar disorder. J Clin Psychiatry. 2010;71(6):e14.

8. McGrath J, Saha S, Welham J, El Saadi O, MacCauley C, Chant D. A systematic review of the incidence of schizophrenia: the distribution of rates and the influence of sex, urbanicity, migrant status and methodology. BMC Med. 2004;28(2):13.

9. Lake CR, Hurwitz N. Schizoaffective disorder merges schizophrenia and bipolar disorders as one disease-there is no schizoaffective disorder. Curr Opin Psychiatry. 2007;20(4):365-79.

10. Malhi GS, Green M, Fagiolini A, Peselow ED, Kumari V. Schizoaffective disorder: diagnostic issues and future recommendations. Bipolar Disord. 2008;10(1 Pt 2):215-30.

11. Keck PE Jr, McElroy SL, Strakowski SM, West SA. Pharmacologic treatment of schizoaffective disorder. Psychopharmacology (Berl). 1994;114(4):529-38.

12. Keck PE Jr, McElroy SL, Strakowski SM. New developments in the pharmacologic treatment of schizoaffective disorder. J Clin Psychiatry. 1996;57(Suppl 9):41-8.

13. Levinson DF, Umapathy C, Musthaq M. Treatment of schizoaffective disorder and schizophrenia with mood symptoms. Am J Psychiatry. 1999;156(8):1138-48.

14. Murru A, Pacchiarotti I, Nivoli AM, Grande I, Colom F, Vieta E. What we know and what we don't know about the treatment of schizoaffective disorder. Eur Neuropsychopharmacol. 2011;21(9):680-90.

15. Svarstad BL, Shireman TI, Sweeney JK. Using drug claims data to assess the relationship of medication adherence with hospitalization and costs. Psychiatr Serv. 2001;52(6):805-11.

16. Doering S, Müller E, Köpcke W, et al. Predictors of relapse and rehospitalization in schizophrenia and schizoaffective disorder. Schizophr Bull. 1998;24(1):87-98.

17. Thompson EE, Neighbors HW, Munday C, Trierweiler S. Length of stay, referral to aftercare, and rehospitalization among psychiatric inpatients. Psychiatr Serv. 2003;54(9):1271-6.

18. Karve S, Cleves MA, Helm M, Hudson TJ, West DS, Martin BC. An empirical basis for standardizing adherence measures derived from administrative claims data among diabetic patients. Med Care. 2008;46(11):1125-33.

19. Leslie R, Gwadry-Sridhar F, Thiebaud P, Patel B. Calculating medication compliance, adherence and persistence in administrative pharmacy claims databases. Pharm Programming. 2008;1:13-9.

20. Martin BC, Wiley-Exley EK, Richards S, Domino ME, Carey TS, Sleath BL. Contrasting measures of adherence with simple drug use, medication switching, and therapeutic duplication. Ann Pharmacother. 2009;43(1):36-44.

21. Stuart B, Davidoff A, Lopert R, Shaffer T, Shoemaker JS, Lloyd J. Does medication adherence lower Medicare spending among beneficiaries with diabetes? Health Serv Res. 2011;46(4):1180-99.

22. Choudhry NK, Shrank WH, Levin RL, et al. Measuring concurrent adherence to multiple related medications. Am J Manag Care. 2009;15(7):457-64.

23. Mazzaglia G, Ambrosioni E, Alacqua M, et al. Adherence to antihypertensive medications and cardiovascular morbidity among newly diagnosed hypertensive patients. Circulation. 2009;120(16):1598-605.

24. Yeaw J, Benner JS, Walt JG, Sian S, Smith DB. Comparing adherence and persistence across 6 chronic medication classes. J Manag Care Pharm. 2009;15(9):728-40.

25. Karve S, Cleves MA, Helm M, Hudson TJ, West DS, Martin BC. Prospective validation of eight different adherence measures for use with administrative claims data among patients with schizophrenia. Value Health. 2009;12(6):989-95.

26. Deyo RA, Cherkin DC, Ciol MA. Adapting a clinical comorbidity index for use with ICD-9-CM administrative databases. J Clin Epidemiol. 1992;45(6):613-9.

27. Karve S, Cleves MA, Helm M, Hudson TJ, West DS, Martin BC. Good and poor adherence: optimal cut-point for adherence measures using administrative claims data. Curr Med Res Opin. 2009;25(9):2303-10.

28. Brook RA, Rajagopalan K, Kleinman NL, Smeeding JE, Brizee TJ, Gardner HH. Incurring greater health care costs: risk stratification of employees with bipolar disorder. Prim Care Companion J Clin Psychiatry. 2006;8(1):17-24.

29. Liu GG, Sun SX, Christensen DB, Zhao Z. Cost analysis of schizophrenia treatment with second-generation antipsychotic medications in North Carolina's Medicaid program. J Am Pharm Assoc. 2007;47(1):77-81.

30. Basu A, Manning WG. Issues for the next generation of healthcare costs analyses. Med Care. 2009;47(7 Suppl 1):S109-14.

31. Wedderburn RWM. Quasi-likelihood functions, generalized linear models, and the Gauss-Newton method. Biometrika. 1974;61:439-47.

32. Manning WG, Mullahy J. Estimating log models: to transform or not to transform? J Health Econ. 2001;20(4):461-94.

33. Robinson D, Woerner MG, Alvir JM, et al. Predictors of relapse following response from a first episode of schizophrenia or schizoaffective disorder. Arch Gen Psychiatry. 1999;56(3):241-7.

34. Frey S, Stargardt T. Performance of compliance and persistence measures in predicting clinical and economic outcomes using administrative data from German sickness funds. Pharmacotherapy. 2012;32(10):880-9.

35. Lang K, Korn J, Muser E, Choi JC, Abouzaid S, Menzin J. Predictors of medication nonadherence and hospitalization in Medicaid patients with bipolar I disorder given long-acting or oral antipsychotics. J Med Econ. 2011;14(2):217-26.

36. Rascati KL, Richards KM, Ott CA, et al. Adherence, persistence of use, and costs associated with second-generation antipsychotics for bipolar disorder. Psychiatr Serv. 2011;62(9):1032-40.

37. Gilmer TP, Dolder CR, Lacro JP, et al. Adherence to treatment with antipsychotic medication and health care costs among Medicaid beneficiaries with schizophrenia. Am J Psychiatry. 2004;161(4):692-9.

38. Sun SX, Liu GG, Christensen DB, Fu AZ. Review and analysis of hospitalization costs associated with antipsychotic nonadherence 
in the treatment of schizophrenia in the United States. Curr Med Res Opin. 2007;23(10):2305-12.

39. Weiden PJ, Kozma C, Grogg A, Locklear J. Partial compliance and risk of rehospitalization among California Medicaid patients with schizophrenia. Psychiatr Serv. 2004;55(8):886-91.

40. Klinkenberg WD, Calsyn RJ. Predictors of receipt of aftercare and recidivism among persons with severe mental illness: a review. Psychiatr Serv. 1996;47(5):487-96.

41. Lin HC, Lee HC. The association between timely outpatient visits and the likelihood of rehospitalization for schizophrenia patients. Am J Orthopsychiatry. 2008;78(4):494-7.

42. Melzer D, Hale AS, Malik SJ, Hogman GA, Wood S. Community care for patients with schizophrenia one year after hospital discharge. BMJ. 1991;303(6809):1023-6.

43. Green JH. Frequent rehospitalization and noncompliance with treatment. Hosp Community Psychiatry. 1988;39(9):963-6.

44. Stickney SK, Hall RC, Garnder ER. The effect of referral procedures on aftercare compliance. Hosp Community Psychiatry. 1980;31(8):567-9.

45. Mitton CR, Adair CE, McDougall GM, Marcoux G. Continuity of care and health care costs among persons with severe mental illness. Psychiatr Serv. 2005;56(9):1070-6.

46. Adair CE, McDougall GM, Mitton CR, et al. Continuity of care and health outcomes among persons with severe mental illness. Psychiatr Serv. 2005;56(9):1061-9.

47. National Institute of Mental Health. What is schizophrenia? 2012. http://www.nimh.nih.gov/health/publications/schizophrenia/ complete-index.shtml\#pub. Assessed 03 Dec 2012.

48. Pfeiffer PN, Ganoczy D, Bowersox NW, McCarthy JF, Blow FC, Valenstein M. Depression care following psychiatric hospitalization in the Veterans Health Administration. Am J Manag Care. 2011;17(9):e358-64.

49. Frank RG, Goldman HH, Hogan M. Medicaid and mental health: be careful what you ask for. Health Aff (Millwood). 2003;22(1):101-13.

50. Johnson N, Oliff P, Williams E. An update on state budget cuts: at least 46 states have imposed cuts that hurt vulnerable residents and cause job loss. Center on Budget and Policy Priorities; 2011. http://www.cbpp.org/cms/?fa=view\&id=1214. Accessed $17 \mathrm{Jul}$ 2013.

51. Thomas MR, Waxmonsky JA, Gabow PA, Flanders-McGinnis G, Socherman R, Rost K. Prevalence of psychiatric disorders and costs of care among adult enrollees in a Medicaid HMO. Psychiatr Serv. 2005;56(11):1394-401.

52. West JC, Rae DS, Huskamp HA, Rubio-Stipec M, Regier DA. Medicaid medication access problems and increased psychiatric hospital and emergency care. Gen Hosp Psychiatry. 2010;32(6):615-22.

53. Hansen RA, Maciejewski M, Yu-Isenberg K, Farley JF. Adherence to antipsychotics and cardiometabolic medication: association with health care utilization and costs. Psychiatr Serv. 2012;63(9):920-8.

54. Kong MC, Nahata MC, Lacombe VA, Seiber EE, Balkrishnan R. Association between race, depression, and antiretroviral therapy adherence in a low-income population with HIV infection. J Gen Intern Med. 2012;27(9):1159-64.

55. Vacek JL, Hunt SL, Shireman T. Hypertension medication use and adherence among adults with developmental disability. Disabil Health J. 2013;6(4):297-302.

56. Wu CH, Erickson SR, Piette JD, Balkrishnan R. The association of race, comorbid anxiety, and antidepressant adherence among Medicaid enrollees with major depressive disorder. Res Social Adm Pharm. 2012;8(3):193-205.

57. Murru A, Pacchiarotti I, Nivoli AM, Colom F, Vieta E. Is schizoaffective disorder still a neglected condition in the scientific literature? Psychother Psychosom. 2012;81(6):389-90.

58. Abouzaid S, Tian H, Zhou H, Kahler KH, Harris M, Kim E. Economic burden associated with extrapyramidal symptoms in a medicaid population with schizophrenia. Community Ment Health J. 2014;50:51-8.

59. Noordsy DL, Phillips GA, Ball DE, Linde-Zwirble WT. Antipsychotic adherence, switching, and health care service utilization among Medicaid recipients with schizophrenia. Patient Prefer Adherence. 2010;4:263-71.

60. Offord S, Lin J, Mirski D, Wong B. Impact of early nonadherence to oral antipsychotics on clinical and economic outcomes among patients with schizophrenia. Adv Ther. 2013;30(3):286-97.

61. Stensland M, Watson PR, Grazier KL. An examination of costs, charges, and payments for inpatient psychiatric treatment in community hospitals. Psychiatr Serv. 2012;63(7):666-71.

62. Stephenson JJ, Tunceli O, Gu T, et al. Adherence to oral secondgeneration antipsychotic medications in patients with schizophrenia and bipolar disorder: physicians' perceptions of adherence vs. pharmacy claims. Int J Clin Pract. 2012;66(6):565-73. 